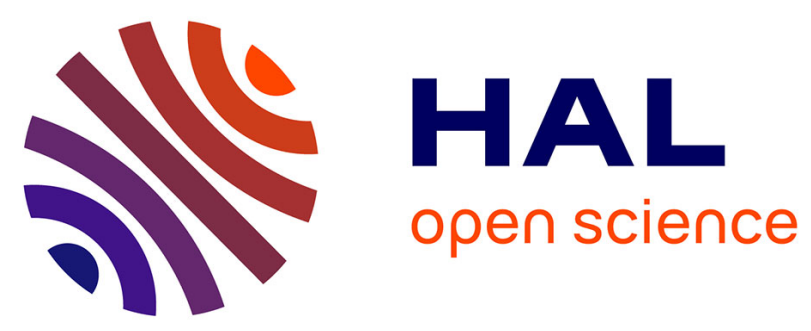

\title{
Direct measurement of the atmospheric neutron flux in the energy range 10-500 $\mathrm{MeV}$
}

Antonio Bonardi, Marco Aglietta, Gianmarco Bruno, Walter Fulgione, Ana Amelia Bergamini Machado

\section{To cite this version:}

Antonio Bonardi, Marco Aglietta, Gianmarco Bruno, Walter Fulgione, Ana Amelia Bergamini Machado. Direct measurement of the atmospheric neutron flux in the energy range 10-500 MeV. Astroparticle Physics, 2010, 34 (4), pp.225. 10.1016/j.astropartphys.2010.07.004 . hal-00679602

\section{HAL Id: hal-00679602 https://hal.science/hal-00679602}

Submitted on 16 Mar 2012

HAL is a multi-disciplinary open access archive for the deposit and dissemination of scientific research documents, whether they are published or not. The documents may come from teaching and research institutions in France or abroad, or from public or private research centers.
L'archive ouverte pluridisciplinaire HAL, est destinée au dépôt et à la diffusion de documents scientifiques de niveau recherche, publiés ou non, émanant des établissements d'enseignement et de recherche français ou étrangers, des laboratoires publics ou privés. 


\section{Accepted Manuscript}

Direct measurement of the atmospheric neutron flux in the energy range 10-500 $\mathrm{MeV}$

Antonio Bonardi, Marco Aglietta, Gianmarco Bruno, Walter Fulgione, Ana Amelia Bergamini Machado

PII: S0927-6505(10)00138-6

DOI: 10.1016/j.astropartphys.2010.07.004

Reference: ASTPHY 1511

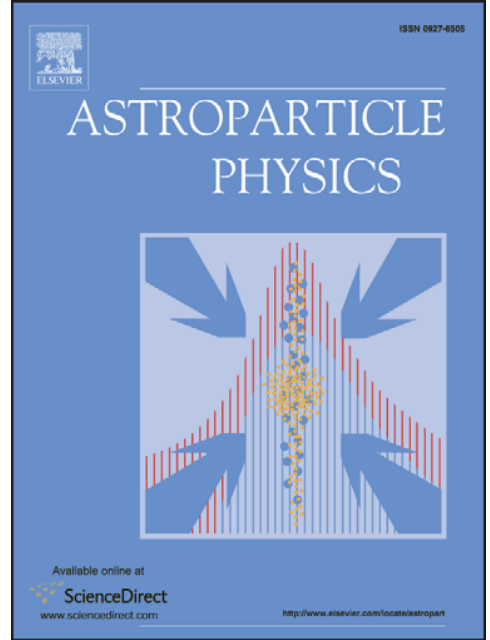

To appear in:

Astroparticle Physics

Received Date:

24 May 2010

Revised Date:

27 July 2010

Accepted Date:

28 July 2010

Please cite this article as: A. Bonardi, M. Aglietta, G. Bruno, W. Fulgione, A.A. Bergamini Machado, Direct measurement of the atmospheric neutron flux in the energy range 10-500 MeV, Astroparticle Physics (2010), doi: 10.1016/j.astropartphys.2010.07.004

This is a PDF file of an unedited manuscript that has been accepted for publication. As a service to our customers we are providing this early version of the manuscript. The manuscript will undergo copyediting, typesetting, and review of the resulting proof before it is published in its final form. Please note that during the production process errors may be discovered which could affect the content, and all legal disclaimers that apply to the journal pertain. 


\title{
Direct measurement of the atmospheric neutron flux in the energy range $10-500 \mathrm{MeV}$
}

\author{
Antonio BONARDI ${ }^{a}{ }^{b}$ ), Marco AGLIETTA ${ }^{b)}$, Gianmarco BRUNO $\left.{ }^{c}\right)^{d}$ ), \\ Walter FULGIONE ${ }^{b}$ ) and Ana Amelia BERGAMINI MACHADO ${ }^{d}$ )
}

\author{
a) Università di Torino, Italy \\ ${ }^{b)}$ INFN and INAF-IFSI Torino, Italy \\ c) Università de L'Aquila, Italy \\ ${ }^{d)}$ INFN-Laboratori Nazionali del Gran Sasso, Italy
}

Abstract

The results of a direct measurement of the atmospheric neutron flux in the energy range $10-500 \mathrm{MeV}$ performed at $42^{\circ} 25^{\prime} 11^{\prime \prime} \mathrm{N}, 13^{\circ} 31^{\prime} 2$ " $\mathrm{E}$, rigidity cutoff $6.3 \mathrm{GV}$, altitude $970 \mathrm{~m}$ a.s.l. (LNGS external site) on November 2008, during minimum solar activity, are reported.

The detector consists of a $1.5 \times 1 \times 1 \mathrm{~m}^{3}$ stainless steel tank filled with 1.2 tons of $0.1 \%$ Gd doped liquid scintillator, monitored by 3 photomultipliers and surrounded by a $4 \pi$ active muon veto. The measurement is performed by observing events formed by two un-vetoed pulses inside a temporal window $95 \mu \mathrm{s}$ long: the first one due to a recoiling proton scattered by a neutron, the second one due to the neutron capture (n, Gd).

The resulting atmospheric neutron fluxes are:

$\Phi(\mathrm{E}>10 \mathrm{MeV})=47 \pm 5$ neutrons $\mathrm{s}^{-1} \mathrm{~m}^{-2}$;

$\Phi(\mathrm{E}>20 \mathrm{MeV})=42 \pm 4$ neutrons $\mathrm{s}^{-1} \mathrm{~m}^{-2}$.

\section{Introduction}

All the neutrons produced by the interactions of galactic and solar cosmic rays in the atmosphere are called "atmospheric neutrons". They are generated in hadronic and electromagnetic air showers by spallation and evaporation processes on Nitrogen and Oxygen nuclei.

Neutrons can be dangerous for electronic devices and human health at aviation altitude where they contribute to about half of the dose equivalent of the secondary cosmic radiation [1],[2],[3], whereas at sea level they are a significant background source for several types of surface detectors. In particular our attention here is focused on studying the atmospheric neutron background for a liquid scintillator antineutrino surface detector.

Since the famous Cowan and Reines experiment [4], nuclear power plants have been used for studying neutrino properties and nowadays neutrino oscillation experimental observations are reactivating this experimental approach.

In principle, it is possible to turn the tide, i.e. to use antineutrino detection for monitoring the activity of nuclear power plants: this may be very useful in particular for detecting illicit or suspicious uses of these facilities [5]. The International Atomic Energy Agency is in the process of developing new technologies 
to monitor nuclear activity on power plants, aiming for non-proliferation safeguards.

The advantages of using $\bar{\nu}_{e}$ for reactor safeguards and monitoring include the availability of real time information on the status of the core, less intrusiveness, and simplified operations from the standpoint of both the reactor operator and the safeguards agency [5].

Such a $\bar{\nu}_{e}$ detector has to be small $\left(\sim 1 \mathrm{~m}^{3}\right)[5]$ and not overburdened, due to cost and logistical reasons: that means a very poor shielding factor for cosmic radiation.

Up to now, $\bar{\nu}_{e}$ detectors are designed to look for Inverse Beta Decay (IBD) $\bar{\nu}_{e}+p \Rightarrow e^{+}+n$ and their active mass is monitored by photomultiplier tubes (PMTs). In case of IBD the prompt $e^{+}$signal is followed by a delayed one due to neutron capture: the double signature allows the disentangling of $\bar{\nu}_{e}$ signals from random background.

Neutrons, in particular atmospheric neutrons, can mimic the IBD reaction. Indeed, they can easily pass through a veto apparatus without interacting, scatter on a proton and then be captured by a nucleus.

The aim of this work is to investigate the atmospheric neutron flux for a $1.5 \mathrm{~m}^{3}$ liquid scintillator detector used as a prototype for a $\bar{\nu}_{e}$ surface detector. The detector was located at $42^{\circ} 25^{\prime} 11^{\prime \prime} \mathrm{N}, 13^{\circ} 31^{\prime} 2$ " E, rigidity cutoff $6.3 \mathrm{GV}$ [6], altitude $970 \mathrm{~m}$ a.s.l, in the LNGS external site.

\section{Detector setup}

The experimental device consists of a parallelepiped stainless tank $(1.5 \mathrm{~m} \mathrm{x} 1 \mathrm{~m}$ $\mathrm{x} 1 \mathrm{~m}$, iron thickness $0.5 \mathrm{~mm}$ ) filled with 1.2 tons of LVD white spirit liquid scintillator [7]. The liquid scintillator was loaded with Gadolinium (Gd) up to $0.1 \%$ fraction mass [8],[9] and monitored by three Photonis 5-inches XP3550B PMTs. The detector is surrounded by a $4 \pi$ active muon veto (see fig. 2 ) which comprises 8 independent modules of plastic scintillator slabs and is globally equipped by twenty seven Photonis 2-inches XP2020 fast PMTs. A general "OR" of all the module outputs produces the veto signal [10].

A TDS5054B 4-channel $300 \mathrm{MHz}$ digital oscilloscope has been used as a waveform digitizer with a $625 \mathrm{MSample} \mathrm{s}^{-1}$ sampling rate. Three oscilloscope channels, set on three different vertical scales, have been used for acquiring the signal resulting from the sum of the 3 PMTs of the detector. The fourth one has been used for acquiring the veto signal. This parallel multi-channel acquisition setup (summarized in fig. 1) allowed us to broaden the total dynamic energy range from 1 up to $500 \mathrm{MeV}$ electron equivalent (hereafter MeVee) while the PMTs noise remained negligible in this energy range.

The waveforms have been acquired after the external trigger which was generated by:

- a random pulse generator for background measurement;

- the occurrence of 3-fold coincidence among detector PMT signals (threshold E 4.5 MeVee, $\Delta t=200 \mathrm{~ns}$, efficiency $\sim 65 \%$ for $\mathrm{E}=5 \mathrm{MeVee}$ and $\sim 1$ for $\mathrm{E} \gtrsim 10 \mathrm{MeVee}$ ), for the atmospheric neutron measurement.

For each acquired waveform the $2.4 \mu \mathrm{s}$ long pre-trigger region is used to evaluate the baseline and its fluctuations for setting the threshold in the after- 


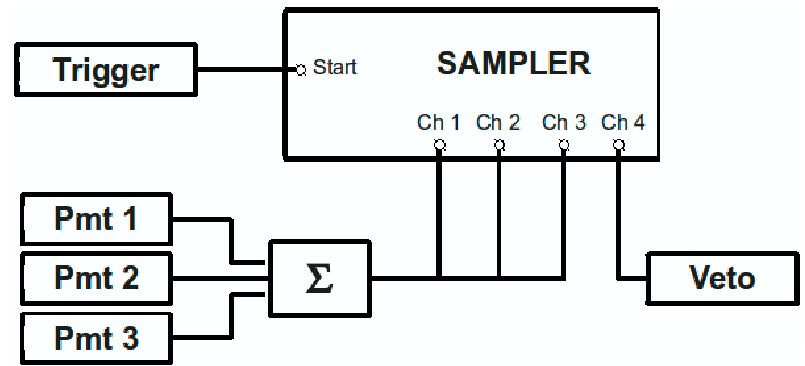

Figure 1: Scheme of the data acquisition setup. The trigger varied according to the scope: it was performed by 1) a random pulse generator for background spectrum measurement or 2 ) the coincidence among the 3 detector PMTs for atmospheric neutron measurement.

trigger region. The threshold is set equal to baseline $+4 \sigma$.

The energy of each pulse was evaluated using the not-saturated oscilloscope channel with larger sensitivity (integration gate $450 \mathrm{~ns}$, total dead time 480 ns). Pulses occurring inside a time window $400 \mathrm{~ns}$ wide, centered at the time of occurrence of the veto signal, were considered "vetoed", otherwise they were considered "un-vetoed".

The aim of the measurement is to observe events with two un-vetoed pulses inside a temporal window $95 \mu \mathrm{s}$ long: the first one due to a recoiling proton scattered by a neutron, the second one due to gammas emitted after the neutron capture (n,Gd).

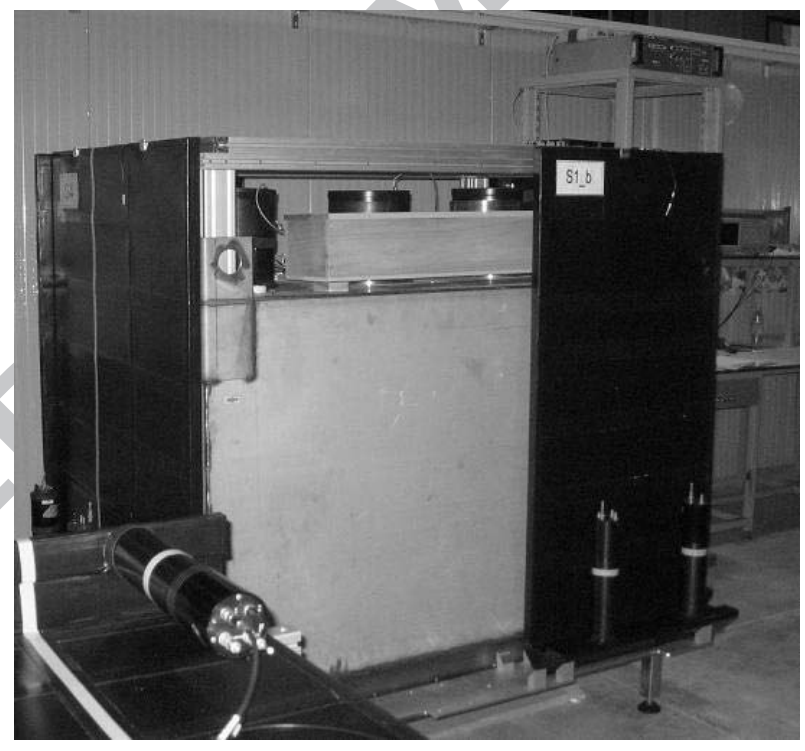

Figure 2: The detector in the veto assembling phase

The absolute energy calibration was done by selecting "vertical" muons by a two plastic scintillator hodoscope. The charge distribution of selected pulses (after rejection of saturated ones) was compared to a Geant4.9.0 based Monte Carlo (MC) simulation of mono-energetic $1 \mathrm{GeV}$ "vertical" muons which esti- 
mated the most probable value of the energy release inside the detector equal to $160 \mathrm{MeV}$. The same MC simulation, conveniently modified, was used for the interpretation of atmospheric neutron data (see sec. 5).

The systematic uncertainty, introduced by this energy calibration technique, has been evaluated taking into account the MC uncertainty and the observed diurnal variation induced by changes in temperature. It is estimated $\sim 2.5 \%$.

\section{The Background}

Placed on-the-ground, the detector is continuously triggered by background particles of different origin, type and energy coming from cosmic radiation and natural radioactivity with a rate $\mathrm{R}(\mathrm{E}>1 \mathrm{MeVee}) \sim 3 \cdot 10^{3} \mathrm{ev} \cdot \mathrm{s}^{-1}$.

The background energy spectrum was measured by acquiring, during 3 days, 230274 randomly triggered waveforms $100 \mu$ s long, corresponding to about 22 seconds real time. In fig. 3 the cumulative background spectrum without applying any veto condition is shown together with the one obtained by selecting only un-vetoed pulses. It can be seen that for energy above $3 \mathrm{MeVee}$, where the component due to radioactivity is absent, the vetoed spectrum is harder due to atmospheric muons going through the counter.

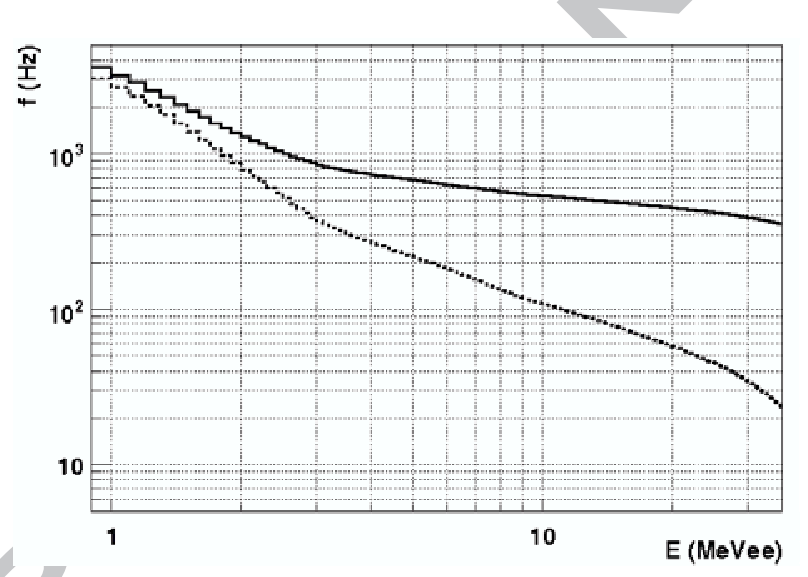

Figure 3: Cumulative background spectra: total spectrum (full line), un-vetoed component (dotted line).

The counting rate of un-vetoed pulses is:

$$
R_{\text {unveto }}(E>10 \mathrm{MeVee})=108 \pm 2(\text { stat. }) \pm 2(\text { syst. }) \mathrm{ev} \cdot \mathrm{s}^{-1}
$$

This value has been used to normalize the observed atmospheric neutron flux described in the next section.

\section{Atmospheric Neutrons}

The atmospheric neutron flux has been measured by acquiring waveforms 100 $\mu \mathrm{s}$ long triggered by the occurrence of 3-fold coincidence among the detector PMTs (energy threshold $\mathrm{E}_{t h} \sim 4.5 \mathrm{MeVee}, \Delta t=200 \mathrm{~ns}$ ). 
During the off-line analysis, the threshold for the prompt pulses was fixed at $5 \mathrm{MeVee}$ (trigger efficiency $\sim 65 \%$ ), while the delayed ones were requested with a time delay respect to the prompt $\Delta t<95 \mu s$ and energy $3 \mathrm{MeVee}<\mathrm{E}<10$ MeVee. Events formed by a prompt pulse and at least one delayed pulse are defined "neutron candidates". Prompt pulses starting a "neutron candidate" are defined "recoil candidates" while the delayed pulses are defined as "neutron capture candidates".

Among 90991 un-vetoed prompt pulses recorded during 5 days of almost continuous data collection we found 8160 "neutron candidates". 2220 of these were expected due to chance coincidences.

By subtracting the chance contribution from the total, taking into account the threshold effect due to the trigger selection and normalizing this result to the measured frequency of un-vetoed background pulses for E $>10 \mathrm{MeVee}$, the observed "neutron candidate" rate is:

$$
\begin{aligned}
& R(E>5 \mathrm{MeVee})=14.62 \pm 0.41(\text { stat. }) \pm 0.96(\text { syst. }) \mathrm{ev}^{-\mathrm{s}^{-1}} \\
& R(E>13 \mathrm{MeVee})=8.40 \pm 0.23(\text { stat. }) \pm 0.30(\text { syst. }) \mathrm{ev} \cdot \mathrm{s}^{-1}
\end{aligned}
$$

In fig. 4 the energy spectrum of "recoil candidates" (gray filled line) can be compared with the energy spectrum of all the un-vetoed prompt pulses (black line).

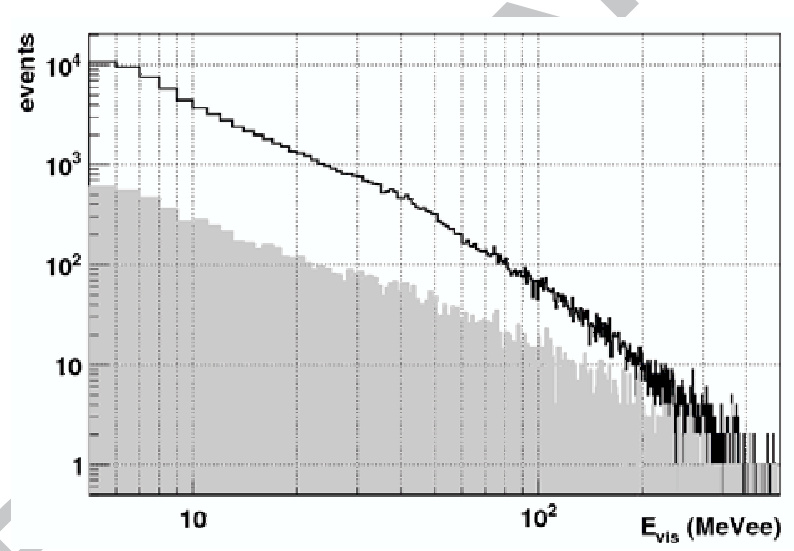

Figure 4: Energy spectra of un-vetoed pulses (black line) and recoil candidates (gray filled line). Both spectra are affected by the threshold effect of trigger selection.

The energy spectrum of "neutron capture candidates" is reported in fig. 5 together with the measured background spectrum renormalized to the same acquisition time. Finally, in fig. 6, the distribution of time differences between delayed and prompt pulses is shown. The slope of the fitted curve is equal to $\tau=24.6 \pm 1.5 \mu \mathrm{s}$, in good agreement with the mean neutron capture time given by Barabanov et al. [9] for neutrons emitted by a ${ }^{252} \mathrm{Cf}$ source placed at the central point of the same detector $(\tau=25 \pm 1 \mu \mathrm{s})$. 


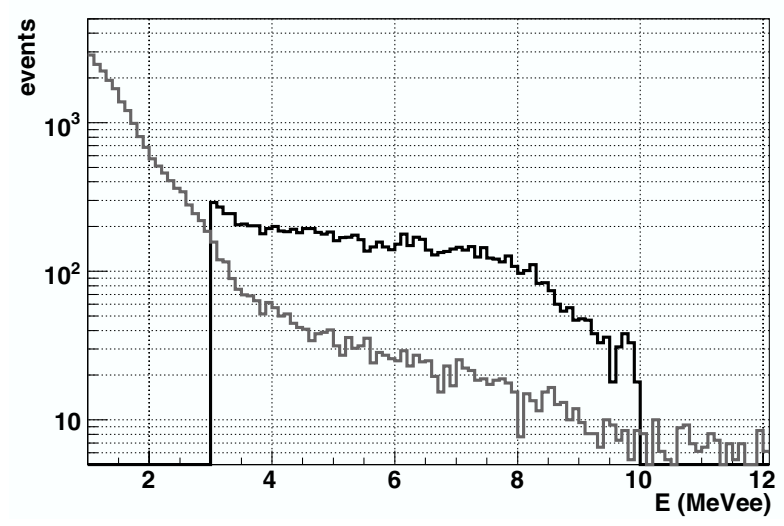

Figure 5: Neutron capture candidates energy spectrum (in black) compared with the renormalized background (in gray).

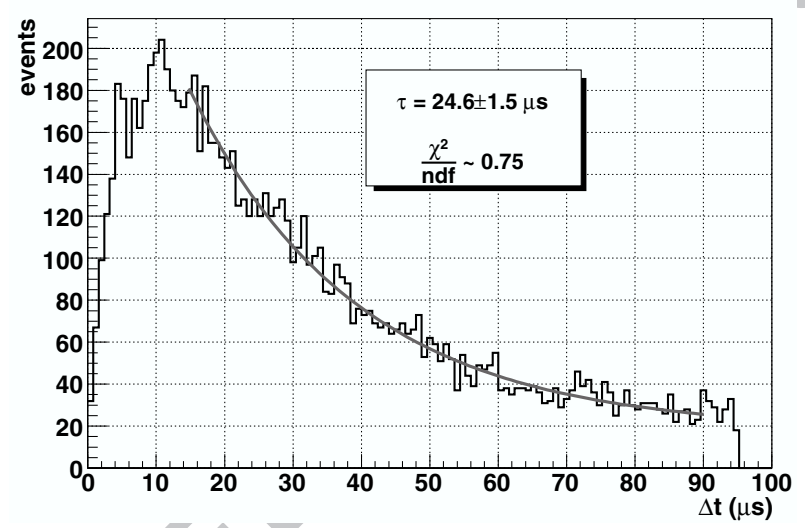

Figure 6: Distribution of time differences between delayed and prompt pulses fitted by an "exponential + constant" function. The slope of the curve is in good agreement with the mean neutron capture time observed by [9] for neutrons emitted by a ${ }^{252} \mathrm{Cf}$ source placed at the central point of the same detector. The constant value is in good agreement with the expected background too.

\section{Discussion}

A Geant4.9.0 based Monte Carlo simulation code has been used for atmospheric neutron data interpretation. The simulation code is the same one used for the energy calibration (see sec. 2).

The goal of this simulation study is to account for:

1. the quenching effect of LVD white-spirit liquid scintillator for recoiling hadrons;

2. the neutron selection efficiency (i.e. the probability for an impinging neutron to give out a detected double signal); 


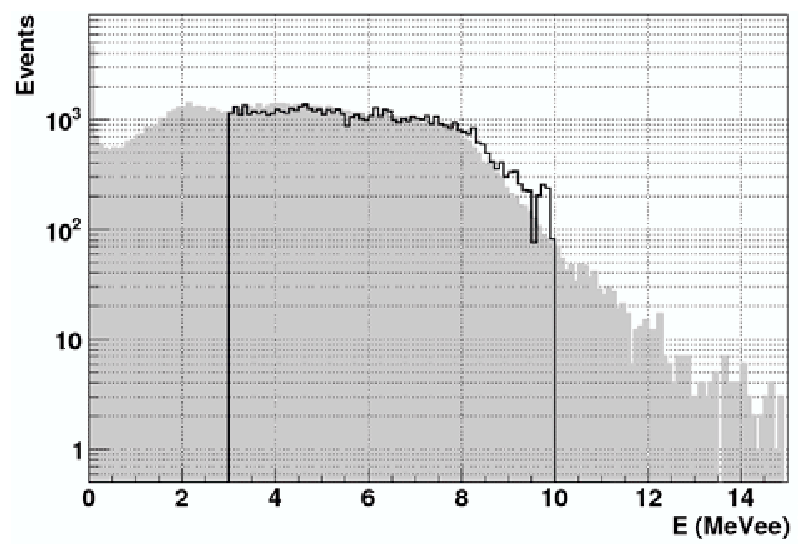

Figure 7: Energy spectrum of neutron capture candidates (black line) compared with the spectrum of simulated homogeneously distributed thermal neutron captures (gray filled line). The peak for $\mathrm{E}<0.2 \mathrm{MeVee}$ is due to detector border effects, while the discrepancy of the two spectra for $\mathrm{E}>8 \mathrm{MeVee}$ is probably due to the starting position of simulated events: since atmospheric neutrons are downward moving, the probability for a delayed neutron capture is not completely homogeneous inside the detector.

10 million neutrons have been simulated over the detector. Their energy was sampled in agreement with the energy spectrum observed at $1020 \mathrm{~m}$ a.s.l. by Kowatari et al. [11] in the Mt. Fuji Area. Their total flux was fixed arbitrarily at $\Phi(\mathrm{E}>20 \mathrm{MeV})=33$ neutrons $\mathrm{s}^{-1} \mathrm{~m}^{-2}$ : this value was obtained by the interpolation of data reported in Tab. 3 by Nakamura et al. [12]. The zenithal distribution of simulated neutrons was $\propto \cos ^{3} \theta$ as reported by Moser et al. [13] while the azimuthal distribution was isotropic: in such a way the detector effective surface is $1.55 \mathrm{~m}^{2}$.

In the simulation code, the concrete walls and roof of the building where the detector is located were neglected because they are located several meters far away from it. That means neutrons absorbed in the concrete are replaced by other neutrons generated in the same concrete by EAS (Extensive Air Shower) whose "not-interacting probability" in the veto system is $\sim 1$.

The quenching factors used for protons, deuterium, tritium and alpha particles are collected in Tab.2, while for heavier particles the quenching factor was assumed equal to 0 and for gammas and electrons equal to 1 [14].

Only neutron events followed by at least one detected neutron capture were selected. The average detection probability of neutron capture in the selection energy window $3 \mathrm{MeVee}<\mathrm{E}<10 \mathrm{MeVee}$ was computed by simulating $1 \cdot 10^{5}$ thermal neutrons $(\mathrm{E}=26 \mathrm{meV})$ homogeneously distributed inside the entire detector volume. The neutron capture detection efficiency is found to be $65.7 \pm 0.3 \%$. The neutron capture energy spectrum for simulated thermal neutrons is shown in fig. 7 together with the spectrum of observed "neutron capture candidates" (after renormalization and background subtraction).

The detector energy resolution is considered in the simulation code while the bin-to-bin migration effect is not included. This effect, which is due to detector inhomogeneities, causes an overestimation in energy of pulses generated in some particular regions of the detector. Thus, the final result appears as an increase 


\begin{tabular}{|c|c|}
\hline Threshold & Counting Rate Increase (\%) \\
\hline $\mathrm{E}_{\text {vis }}>3$ MeVee & $33 \%$ \\
$\mathrm{E}_{\text {vis }}>5$ MeVee & $18 \%$ \\
$\mathrm{E}_{\text {vis }}>10$ MeVee & $15 \%$ \\
$\mathrm{E}_{\text {vis }}>13$ MeVee & $11.5 \%$ \\
$\mathrm{E}_{\text {vis }}>15$ MeVee & $10 \%$ \\
\hline
\end{tabular}

Table 1: "Bin-to-bin migration " effect on background un-vetoed pulses counting rates. This effect is due to the detector inhomogeneities and is inversely proportional to the energy threshold.

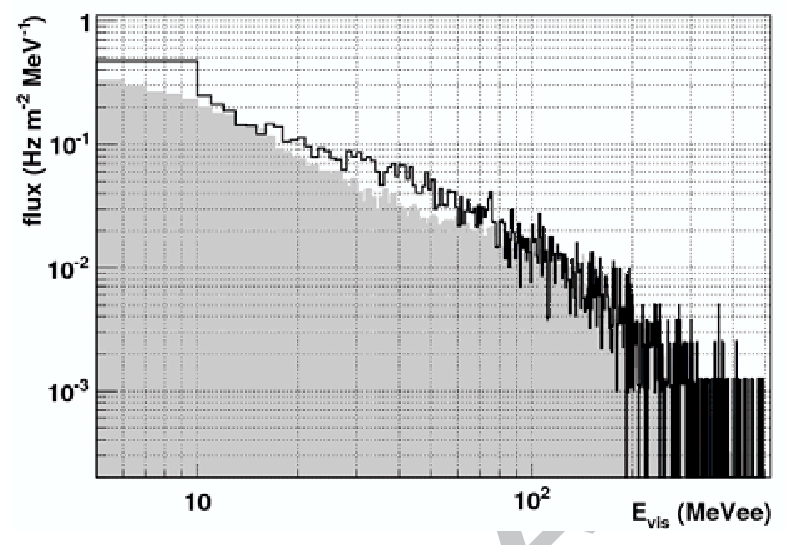

Figure 8: Differential visible energy spectra: observed recoil candidates after background subtraction (black line), simulated ones (gray filled line). In the energy range $5 \mathrm{MeVee}<\mathrm{E}_{\text {vis }}<$ $10 \mathrm{MeVee}$, the experimental data are plotted in a single bin because the trigger efficiency effect did not allow a better resolution. N.B.: the experimental data are affected by the bin-to-bin migration effect.

in the high energy counting rate. This effect on a LVD counter was studied by Porta [15] and can be parameterized as reported in Tab. 1. The bin-to-bin migration effect has been considered in the calculation of the neutron flux.

In fig. 8-9 the differential and cumulative energy spectra of observed recoil candidates (after background subtraction and normalization to the trigger threshold efficiency in the energy range $5 \mathrm{MeVee}<\mathrm{E}_{v i s}<10 \mathrm{MeVee}$ ) and simulated ones are shown.

The counting rate of observed recoil candidates has been compared to the simulated one for $\mathrm{E}_{v i s}>5$ and $13 \mathrm{MeVee}$, corresponding to neutron energy $>10$ and $20 \mathrm{MeV}$ (see Tab. 2). By applying the correction for the bin-to-bin migration effect to the experimental data, the absolute neutron flux for energy above 10 and $20 \mathrm{MeV}$ was found to be:

$$
\begin{aligned}
& \Phi(E>10 M e V)=47 \pm 5 \text { neutrons } s^{-1} m^{-2} \\
& \Phi(E>20 M e V)=42 \pm 4 \text { neutrons } s^{-1} m^{-2}
\end{aligned}
$$

The uncertainties on the reported fluxes were computed considering statistical and systematic errors. The statistical uncertainties were due to the Poissonian 


\section{ACCEPTED MANUSCRIPT}

6 CONCLUSION

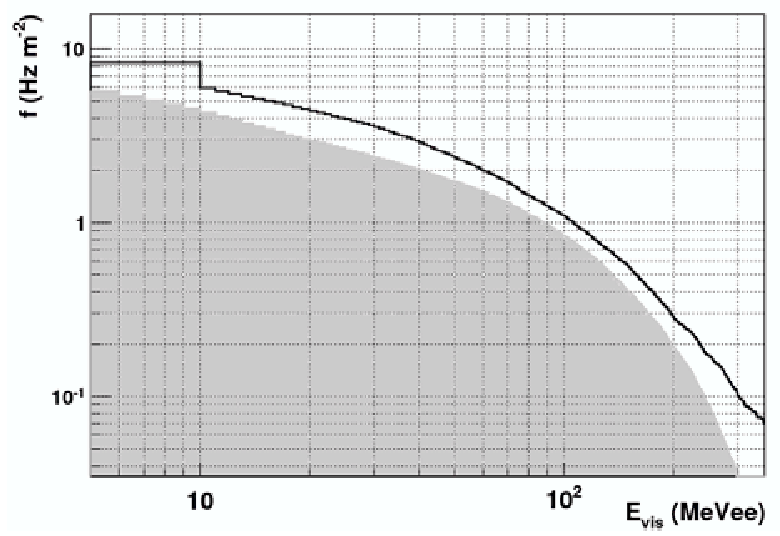

Figure 9: Cumulative visible energy spectra: recoil candidates after background subtraction (black line), simulated ones (gray filled line). N.B.: the experimental data are affected by the bin-to-bin migration effect.

fluctuations of the observed and simulated counting rates inside our detector, whilst the systematic uncertainties were due to the energy calibration of the detector and the applied quenching factor reported in Tab. 2 .

\begin{tabular}{|c|c|c|c|c|}
\hline Kin. Energy $(\mathrm{MeV})$ & protons & alphas & deuterons & tritiums \\
\hline 1.0 & 0.230 & 0.020 & 0.150 & 0.1100 \\
2.0 & 0.310 & 0.040 & 0.220 & 0.1800 \\
4.0 & 0.390 & 0.080 & 0.270 & 0.2300 \\
6.0 & 0.450 & 0.100 & 0.320 & 0.2600 \\
8.0 & 0.500 & 0.120 & 0.350 & 0.2900 \\
10.0 & 0.534 & 0.140 & 0.380 & 0.3100 \\
12.0 & 0.565 & 0.155 & 0.412 & 0.3330 \\
16.0 & 0.625 & 0.180 & 0.466 & 0.3600 \\
20.0 & 0.664 & 0.202 & 0.520 & 0.3900 \\
28.0 & 0.750 & 0.248 & 0.592 & 0.4400 \\
30.0 & 0.764 & 0.260 & 0.620 & 0.4500 \\
40.0 & 0.850 & 0.314 & 0.710 & 0.5100 \\
58.0 & 0.950 & 0.386 & 0.810 & 0.6000 \\
70.0 & 0.980 & 0.435 & 0.870 & 0.6500 \\
90.0 & 1.000 & 0.515 & 0.930 & 0.7400 \\
100.0 & 1.000 & 0.550 & 0.944 & 0.7800 \\
\hline
\end{tabular}

Table 2: Quenching factor for protons, deuterons, tritiums, alphas in white-spirit liquid scintillator [14].

\section{Conclusion}

On November 2008 during solar minimum activity, a detector filled with 1.2 tons of $0.1 \% \mathrm{Gd}$ doped liquid scintillator, supplied with an active muon veto and located at $42^{\circ} 25^{\prime} 11^{\prime \prime} \mathrm{N}, 13^{\circ} 31^{\prime} 2^{\prime \prime}$ E, rigidity cutoff $6.3 \mathrm{GV}$ [6], altitude 
$970 \mathrm{~m}$ a.s.l., was used to perform a measurement of the atmospheric neutron absolute flux. Our detector is not able to observe fast fluctuations of the atmospheric neutron flux (e.g. the Forbush Decrease) because that was out of our goals. In any case, it would be sufficient to modify the DAQ setup for making our detector sensitive to such fluctuations and turning it into a Atmospheric Neutron Monitor. The aim of the measurement is to observe the energy released by protons scattered by neutrons which are subsequently captured. We obtained the following atmospheric neutron fluxes:

$$
\begin{aligned}
& \Phi(E>10 M e V)=47 \pm 5 \text { neutrons } s^{-1} m^{-2} ; \\
& \Phi(E>20 M e V)=42 \pm 4 \text { neutrons } s^{-1} m^{-2} .
\end{aligned}
$$

Our results are $\sim 30 \%$ larger than the one reported by Kowatari et al. [11] and Nakamura et al. [12] at similar altitude in Japan in the Mt. Fuji area, $35^{\circ}$ $22^{\prime} \mathrm{N}, 138^{\circ} 43^{\prime}$ E, rigidity cutoff $10.43 \mathrm{GV}$, altitude $1020 \mathrm{~m}$ a.s.l. This broad difference could be easily explained by the different rigidity cutoff. This effect has been already observed and reported by Goldhagen et al. in Fig. 7 [1]. The same figure clearly shows that, even if the total neutron flux changes with the rigidity cutoff, the shape of its energy spectrum still remains almost the same [1]. A secondary role could be also played by the different period in the solar activity cycle because Kowatari et al. performed their measurement on September 2002, i.e. during solar maximum activity.

The measurements reported by Goldhagen et al. [1], Kowatari et al. [11] and Nakamura et al. [12] have been performed by using a "Bonner multi-sphere neutron spectrometer" and the acquired data were un-folded by a simulation code (SAND II [16]) with an initial estimated spectrum.

The present measurement was almost calorimetric and did not depend strictly on the "a priori" spectrum. In our knowledge this is the only calorimetric measurement of atmospheric neutron flux.

\section{References}

[1] P. Goldhagen et al. (2002) "Measurement ofthe energy spectrum of cosmicray induced neutrons aboard an ER-2 high-altitude airplane", NIM A 476, 4251

[2] S. Roesler, W. Heinrich, H. Schraube (1998), Radiat. Res. 149, 87

[3] A. Kurochkin et al. (1999), Radiat. Prot. Dosim. 83, 281

[4] C. L. Cowan, Jr., F. Reines, F. B. Harrison, H. W. Kruse and A. D. McGuire (1956) "Detection of the Free Neutrino: A Confirmation", Science 124, 103

[5] A. Bernstein et al. (2002) "Nuclear reactor safeguards and monitoring with antineutrino detectors", Journ. Appl. Phys. Vol 91, N. 7

[6] S. Cecchini et al. (2005), "On the altitude dependence of gamma ray spectra in the Earth's atmosphere", $29^{\text {th }}$ International Cosmic Ray Conference Pune 2, 349-352 
[7] G. Bari et al. (1989) "The large-volume detector (LVD) : a multipurpose underground detector at Gran Sasso", NIM A 277, 11-16

[8] N. A. Danilov et al. (2007), Radiochemistry 49, 281

[9] I. R. Barabanov et al. , on printing on JINST

[10] A. Bonardi phD Thesis (2010), "Study of Cosmic Ray Neutrons"

[11] M. Kowatari, et al. (2005), "Evaluation of the Altitude Variation of the Cosmic-ray Induced Enviromental Neutrons in the Mt. Fuji Area" Journal of Nuc. Sc. and Tec., Vol.42,No.6, 495-502

[12] T. Nakamura, et al. (2005), Sequential Measurements of Cosmic-Ray Neutron Spectrum and Dose Rate at See Level in Sendai, Japan Journal of Nuc. Sc. and Tec., Vol.42,No.10, 843-853

[13] M. R. Moser et al. (2005), "Atmospheric Neutron Measurements in the 10-170 MeV Range”, 29 ${ }^{\text {th }}$ International Cosmic Ray Conference Pune 2, $421-424$

[14] O. Ryaskaya, private comunication

[15] A. Porta phD Thesis (2005), "Energy measurement in LVD to reconstruct Supernova neutrino emission"

[16] W. N. McElroy et al. (1967), "A computer-automated Iterative Method for Neutron Flux Spectra Determination by Foil Activation", AFWL-TR-67-41, Air Force Weapons Laboratory 